\title{
ANÁLISE DO USO COMBINADO DE PARÂMETROS DE CALIBRAÇÃO CONVENCIONAIS E DE CALIBRAÇÃO EM SERVIÇO NA AEROTRIANGULAÇÃO DE AEROFOTOS OBTIDAS COM CÂMERA DE BAIXO CUSTO
}

An analysis of the combined use of conventional calibration parameters and calibration on job in the aerotriangulation of aerial phfotographs taken from lowcost camara

\author{
JUILSON J. JUBANSKI ${ }^{1}$ \\ EDSON A. MITISHITA ${ }^{2}$ \\ HANS-PETER BÄHR ${ }^{1}$ \\ ${ }^{1}$ KIT - Karlsruhe Institut of Technology \\ IPF - Institut für Photogrammetrie und Fernerkundung \\ Kaiserstraße 1276131 Karlsruhe - Deutschland \\ \{juilson.jubanski, hans-peter.baehr\}@ipf.uni-karlsruhe.de \\ ${ }^{2}$ Universidade Federal do Paraná \\ CPGCG - Curso de Pós Graduação em Ciências Geodésicas \\ Caixa Postal 19.001 CEP 81531-990 Curitiba, Brasil \\ mitishita@ufpr.br
}

\begin{abstract}
RESUMO
O interesse pela utilização de câmeras digitais de pequeno formato e baixo custo vem continuamente aumentando na aerofotogrametria, como uma alternativa viável para mapeamento de pequenas áreas. Muitas pesquisas estão sendo realizadas a respeito da qualidade geométrica que essas câmeras permitem alcançar, e um ponto fundamental nessas pesquisas é a calibração desses equipamentos. A estabilidade destes equipamentos tem sido alvo de intensas pesquisas. Apesar de os parâmetros de calibração se mostrarem constantes ao longo do tempo, as diferenças das condições ambientais durante a calibração e durante o vôo fotogramétrico podem gerar erros não-modelados pela calibração clássica. Entre as câmeras fotogramétricas digitais de grande formato, a utilização de parâmetros de calibração em serviço é padrão nas aerotriangulações. Este trabalho apresenta uma análise da inclusão desses parâmetros na aerotriangulação de um bloco de 13 imagens tomadas
\end{abstract}


com uma câmera de baixo custo. Os resultados apontam para uma melhoria significativa no ajustamento quando da aplicação desses parâmetros, principalmente na altimetria.

Palavras-chave: Fotogrametria; Calibração de Câmeras; Parâmetros Adicionais.

\section{ABSTRACT}

The interest in the use of small format and low-cost digital cameras is increasing in aerial Photogrammetry. This is as a viable alternative for small areas mapping. A great deal of research on the geometric quality that those cameras allow are currently being carried out and a crucial issue is the system calibration. The stability of these equipment is target of intense research. In spite of the constant calibration parameters throughout the time, differences in the environmental conditions during calibration and during the photogrammetric flight may issue errors not treated in classical calibration. Among the large frame photogrammetric digital cameras, the use of on-the-job calibration parameters is currently standard for the aerotriangulation. This paper analyses the inclusion of those parameters in the aerotriangulation of a block of 13 images taken with a low-cost digital camera. The results show a significant accuracy improvement when those parameters are used, mainly in altimetry.

Keywords: Photogrammetry; Camera Calibration; Additional Parameters.

\section{INTRODUÇÃO}

A utilização de câmeras fotográficas digitais de baixo-custo na aerofotogrametria vem sendo discutida intensamente nos últimos anos. $\mathrm{O}$ principal questionamento a respeito do uso desses equipamentos diz respeito à estabilidade dos parâmetros de calibração (Läbe et. al., 2004), obtidos principalmente por técnicas de fotogrametria a curta-distância; ao longo do tempo e durante o vôo fotogramétrico. Habib et. al. (2005). Habib et. al., (2006) prova a estabilidade dos parâmetros de calibração das câmeras de baixo custo ao longo do tempo; mesmo assim a qualidade de trabalhos aerofotogramétricos realizados com essas câmeras continua sendo questionada.

Com o aumento expressivo do uso de câmeras métricas digitais de grande formato - as quais são compostas em sua maioria por diversos sistemas sensores de médio-formato acoplados - a calibração por métodos a curta distância tornou-se um procedimento padrão na aerofotogrametria (Jubanski et. al., 2009). No entanto, os resultados das aerotriangulações de blocos de imagens, tomadas com essas câmeras, só são satisfatórios quando se lança mão da utilização de parâmetros de calibração em serviço (Cramer, 2006). O principal objetivo deste trabalho é apresentar uma metodologia análoga para a utilização destes parâmetros na execução de aerotriangulação de aerofotos obtidas com câmeras digitais de baixo custo. Para tanto, os resultados obtidos com o modelo clássico de aerotriangulação serão 
comparados com os resultados obtidos com a metodologia proposta, empregando análises estatísticas das discrepâncias observadas em pontos de verificação no espaço-objeto (referencial geodésico). Por fim, são apresentadas considerações sobre os resíduos obtidos na aerotriangulação, a fim de se verificar a existência de alguma tendência sistemática quando de aplicam os parâmetros de calibração em serviço.

Este trabalho é organizado da seguinte maneira: Na seção 2 a metodologia utilizada é apresentada, juntamente com a revisão de literatura e estado da arte. $\mathrm{Na}$ seção 2.1 trata-se da orientação interna das imagens, na seção 2.2 trata-se da modelagem escolhida para os parâmetros adicionais e na seção 2.3 é apresentada a metodologia de coleta dos pontos de apoio e verificação a partir de dados LIDAR. $\mathrm{Na}$ seção 3 são apresentados os resultados e análises estatísticas. A seção 3.1 apresenta os resultados no espaço objeto e a seção 3.2 as analises de resíduos das aerotriangulações. Por fim a seção 4 apresenta as conclusões e recomendações.

\section{METODOLOGIA}

A metodologia adotada consiste de três etapas fundamentais: a primeira é a orientação interna das aerofotos, a segunda o processamento da aerotriangulação (orientação externa) e por final as analises estatísticas.

\subsection{Orientação Interna}

O propósito tradicional da calibração de câmeras fotogramétricas é tornar possível, através de modelos matemáticos, a reconstrução da geometria projetiva do feixe de raios de luz que entra na câmera no momento da exposição, a partir das coordenadas bi-dimensionais medidas nas imagens deformadas (Cramer, 2006). Os principais parâmetros de calibração, segundo essa visão clássica, são a distância focal calibrada (c); as coordenadas do ponto principal $\left(\mathrm{x}_{0}, \mathrm{y}_{0}\right)$; os parâmetros de distorção radial simétrica $\left(\mathrm{k}_{1}, \mathrm{k}_{2}, \mathrm{k}_{3}\right)$, causada pela deficiência no processo de fabricação das lentes de uma objetiva; e os parâmetros da distorção descentrada $\left(\mathrm{P}_{1}, \mathrm{P}_{2}\right)$, causada pelo não alinhamento dos eixos ópticos das lentes que compõem a objetiva. Este conjunto de parâmetros será denominado "parâmetros de calibração convencionais" ao longo deste trabalho. Um fator a ser ressaltado nos processos de calibração de campo, com a utilização, por exemplo, de fotos verticais, é a forte correlação estatística existente entre os parâmetros de orientação interior $\left(\mathrm{c}, \mathrm{x}_{0}, \mathrm{y}_{0}\right.$, $\left.\mathrm{k}_{1}, \mathrm{k}_{2}, \mathrm{k}_{3}, \mathrm{P}_{1}, \mathrm{P}_{2}\right)$ e os parâmetros de orientação exterior $\left(\kappa, \varphi, \omega, \mathrm{X}_{0}, \mathrm{Y}_{0}, \mathrm{Z}_{0}\right)$. Por esse motivo, não se pode realizar uma calibração sem o emprego de técnicas que permitam quebrar esta correlação, como é o caso do método das Câmeras Convergentes ou dos Campos Mistos. Atualmente, o método das câmeras convergentes, apresentado em Brown (1969), é o procedimento mais usual para a calibração de câmeras digitais de baixo custo. Os resultados obtidos na calibração 
da câmera Sony DSC-F717, utilizada nesse trabalho, são apresentados em De Lara et. al. (2004).

O processo de pré-correção dos erros sistemáticos, o qual visa à recuperação da geometria projetiva de uma observação na imagem digital, pode ser realizado em duas etapas principais: a primeira é a transformação da observação, feita no referencial levogiro de imagem (linha e coluna), para um referencial dextrogiro (em $\mathrm{mm}$ ) com a origem no centro da imagem. A segunda etapa é a correção das distorções de lentes, através da aplicação dos parâmetros de calibração sobre as coordenadas originais de imagem $\left(\mathrm{x}_{\mathrm{i}}, \mathrm{y}_{\mathrm{i}}\right)$. Na equação (1) abaixo, $\delta \mathrm{r}\left(\mathrm{k}_{1,2,3}\right)$ e $\delta \mathrm{d}\left(\mathrm{P}_{1,2}\right)$ representam a correção das distorções radial simétrica e descentrada, respectivamente.

$$
\left\{\begin{array}{l}
\mathrm{x}_{\mathrm{f}}=\mathrm{x}_{\mathrm{i}}+\mathrm{x}_{0}+\delta \mathrm{r}_{\mathrm{x}}\left(\mathrm{k}_{1,2,3}\right)+\delta \mathrm{d}_{\mathrm{x}}\left(\mathrm{P}_{1,2}\right) \\
\mathrm{y}_{\mathrm{f}}=\mathrm{y}_{\mathrm{i}}+\mathrm{y}_{0}+\delta \mathrm{r}_{\mathrm{y}}\left(\mathrm{k}_{1,2,3}\right)+\delta \mathrm{d}_{\mathrm{y}}\left(\mathrm{P}_{1,2}\right)
\end{array}\right.
$$

Os valores $\left(\mathrm{x}_{\mathrm{f}}, \mathrm{y}_{\mathrm{f}}\right)$ são as coordenadas refinadas, referidas ao referencial fotogramétrico, a serem utilizadas durante o processamento da aerotriangulação, e $\left(\mathrm{x}_{0}, \mathrm{y}_{0}\right)$ são as coordenadas do ponto principal, obtidas na calibração.

\subsection{Parâmetros Adicionais e Calibração em Serviço}

Ackermann (1981) demonstra a impossibilidade de se eliminar todos os erros sitemáticos de uma camera utlizando-se somente dos parâmetros convencionais de calibração. Jacobsen (2001) afirma que outros modelos matemáticos empíricos podem ser utilizados num processo de calibração em serviço, visando otimizar o processo de recuperação da geometria projetiva, tendo em vista, por exemplo, os efeitos provocados pela não-correção da refração fotogramétrica para a atmosfera real, existente no instante da tomada das aerofotos; a utilização da câmara em condições distintas entre o instante da calibração e da tomada das aerofotos (pressão, temperatura, umidade e trepidação) e por fim pela não-estabilidade dos parâmetros determinados no instante da calibração.

A preocupação com a estabilidade das câmeras digitais de baixo custo tem grande relevância para uma aerofotogrametria de "baixo custo", pois essas câmeras não são especialmente projetadas para a realização de trabalhos aerofotogramétricos. Pesquisa realizada por Habib et. al., (2006), mostra que os parâmetros de calibração apresentam boa estabilidade no intervalo de tempo entre calibrações realizadas, entretanto a estabilidade do sensor no ambiente e no instante da tomada das imagens aéreas não foi tratada. Esse questionamento já existe desde a época das câmeras analógicas de grande formato e muitos estudos foram realizados nessa área, como por exemplo Ebner (1976), Grün (1978), Jacobsen (1982), Moniwa (1981) e mais recentemente por Habib (2002). No entanto, para aquelas

Bol. Ciênc. Geod., sec. Artigos, Curitiba, v. 16, nº 4, p.503-518, out-dez, 2010. 
câmeras, dada a grande estabilidade geométrica dos parâmetros que recuperam a geometria projetiva, como também as mínimas distorções no sistema de lentes, permitiam, em muitas aplicações, que muitas das correções de erros sistemáticos pudessem ser negligenciadas. Para as câmeras digitais de baixo custo, entretanto, é de se esperar que uma modelagem aprimorada dos efeitos que afetam a geometria projetiva traga melhorias significativas nos resultados das aerotriangulações. Segundo Dörstel (2007), a realização de aerotriangulações calibração em serviço já é um padrão entre as câmeras digitais de grande formato com a combinação de vários sensores, por causa de erros sistemáticos introduzidos na geração de imagens virtuais. Marcato (2008) apresenta uma série de experimentos para aerotriangulação com calibração em serviço. O presente trabalho apresenta a modelagem matemética completa e a avaliação de um procedimento de calibração em serviço para uma câmera digital de pequeno formato e baixo custo.

Existe uma confusão na literatura sobre o conceito de auto-calibração (Clark et. al, 1998). Brown (1989) considera auto-calibração um procedimento de calibração no qual nenhum tipo de controle é necessário. Entretanto, na prática, isso se mostra impraticável, e por essa razão, usa-se o termo auto-calibração quando a determinação de parâmetros adicionais se dá durante o ajustamento do bloco. Esses parâmetros visam uma compensação de erros não tratados durante o processo de pré-refinamento das coordenadas (Jacobsen, 2001). Conceitualmente, o processo de calibração apresentado por Brown (1969) é, também, um processo de autocalibração no qual erros sistemáticos (distorções radial simétrica e descentrada) são parametrizados. Neste trabalho, o termo "calibração em serviço" (on-the-job calibration) será usado para evitar a confusão entre calibração convencional e ajustamento em bloco com auto-calibração, após o pré-refinamento das coordenadas. Para a modelagem matemática de uma aerotriangulação com calibração em serviço exige-se a ortogonalidade (independência estatística) entre os parâmetros de orientação exterior (posição tridimensional do centro perspectivo no espaço-imagem e suas 3 rotações eulerianas), e os parâmetros adicionais (Kraus, 1996). Desta forma, a correlação estatística entre esses dois grupos de parâmetros é, por definição, zero. Kupfer et. al., (1982) apresenta uma análise rigorosa sobre correlações am ajustamentos de feixe de raios. Ebner (1976) apresenta um conjunto de 24 componentes de distorção envolvendo 12 parâmetros não-correlacionados a serem aplicados nas aerotriangulações (Fig. 1). Neste trabalho, esse conjunto de parâmetros será denominado "parâmetros calibração em serviço". 
Figura 1 - Parâmetros Adicionais de Auto-Calibração propostos por Ebner (1976).

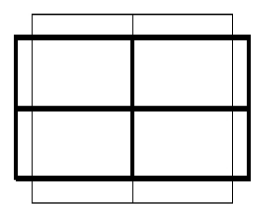

$\delta \mathrm{a}_{\mathrm{x}}=+\mathrm{b}_{1} \mathrm{x}$

$\delta a_{y}=-b_{1} y$

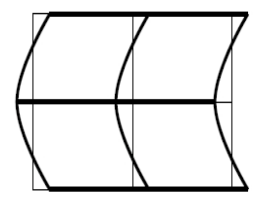

$+\mathrm{b}_{5}\left(\mathrm{y}^{2}-2 \mathrm{~B}_{\mathrm{y}}^{2} / 3\right)$

0

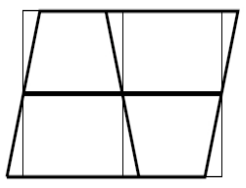

$+b_{9} y\left(x^{2}-2 B_{x}^{2} / 3\right)$

0



$+\mathrm{b}_{2} \mathrm{y}$

$+\mathrm{b}_{2} \mathrm{x}$

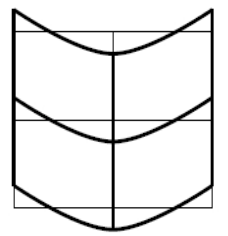

0

$+\mathrm{b}_{6}\left(\mathrm{x}^{2}-2 \mathrm{~B}_{\mathrm{x}}^{2} / 3\right)$

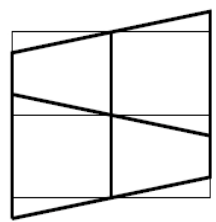

$$
\begin{array}{ccc}
0 & +b_{11}\left(x^{2}-2 B_{x}^{2} / 3\right)\left(y^{2}-2 B_{y}^{2} / 3\right) & 0 \\
+b_{10} x\left(y^{2}-2 B_{y}^{2} / 3\right) & 0 & +b_{12}\left(x^{2}-2 B_{x}^{2} / 3\right)\left(y^{2}\right.
\end{array}
$$
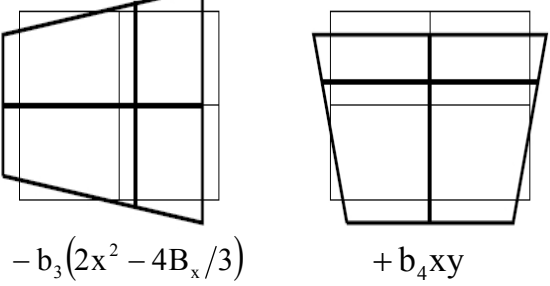

$+\mathrm{b}_{4} \mathrm{xy}$

$+b_{3} x y$
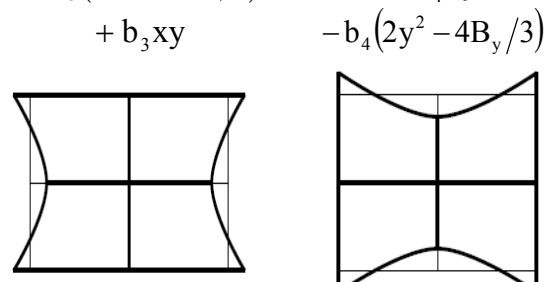

$+b_{7} x\left(y^{2}-2 B_{y}^{2} / 3\right)$

0

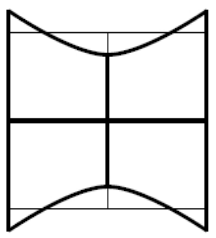

0

$+\mathrm{b}_{8} \mathrm{y}\left(\mathrm{x}^{2}-2 \mathrm{~B}_{\mathrm{x}}^{2} / 3\right)$

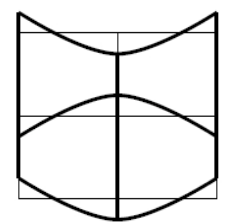

Fonte: Adaptado de (Ebner, 1976)

Nestas equações, $(\mathrm{x}, \mathrm{y})$ representam as coordenadas refinadas no referencial fotogramétrico, c é a distância focal, $b_{1} a b_{12}$ são os 12 parâmetros de Ebner e $B_{x} e$ $\mathrm{B}_{\mathrm{y}}$ as dimensões da imagem (ou o número de linhas e colunas). Para cada observação fotogramétrica são adicionados os termos $\delta \mathrm{a}_{\mathrm{x}}$ e $\delta \mathrm{a}_{\mathrm{y}}$. Por esta razão esses parâmetros são chamados "adicionais", pois estes são "adicionados" no equacionamento da aerotriangulação. Esses dois termos $\left(\delta \mathrm{a}_{\mathrm{x}} \mathrm{e} \delta \mathrm{a}_{\mathrm{y}}\right)$ são a combinação das componentes de distorção apresentadas na Fig. 1. $O$ equacionamento final é apresentado em (2):

$$
\left\{\begin{array}{l}
x=-c \cdot \frac{D X}{D Z}+\delta a_{x} \\
y=-c \cdot \frac{D Y}{D Z}+\delta a_{y}
\end{array}\right.
$$

Bol. Ciênc. Geod., sec. Artigos, Curitiba, v. 16, nº 4, p.503-518, out-dez, 2010. 
Onde:

$$
\left\{\begin{array}{l}
D X=m_{11}\left(X-X_{0}\right)+m_{12}\left(Y-Y_{0}\right)+m_{13}\left(Z-Z_{0}\right) \\
D Y=m_{21}\left(X-X_{0}\right)+m_{22}\left(Y-Y_{0}\right)+m_{23}\left(Z-Z_{0}\right) \\
D Z=m_{31}\left(X-X_{0}\right)+m_{32}\left(Y-Y_{0}\right)+m_{33}\left(Z-Z_{0}\right)
\end{array}\right.
$$

Nas equações (3), (X,Y,Z) são as coordenadas do ponto no espaço objeto, $\left(\mathrm{X}_{0}, \mathrm{Y}_{0}, \mathrm{Z}_{0}\right)$ as coordenadas do centro perspectivo da câmera e $\mathrm{m}_{11}$ a $\mathrm{m}_{33}$ os elementos da matriz de rotações. De forma semelhante ao que se faz durante uma calibração convencional, os parâmetros de calibração em serviço entram no sistema como sendo variáveis e têm o seus valores injuncionados para zero no caso de serem não-significativos. Através da analise da matriz variância-covariância dos parâmetros ajustados, juntamente com um teste estatístico adequado, é possível ver a significância dos parâmetros determinados no ajustamento e a utilização dos mais significativos. Maiores detalhes na seção 3.

\subsection{Pontos de Apoio e de Verificação}

Neste trabalho foram utilizados pontos pré-sinalizados (Wandresen, 2004), cujas coordenadas foram extraídas de um levantamento Laser-Scanner de 05/09/2003, realizado com o sensor Optech ALTM 2050, de propriedade do Lactec (Instituto de Tecnologia para o Desenvolvimento). O uso de dados LIDAR ("Light Detection and Ranging") como apoio para trabalhos fotogramétricos têm um grande potencial, conforme demonstrado em De Lara et. al. (2006) e em Habib et. al. (2004). Pelo fato de os pontos pré-sinalizados não terem distribuição geométrica suficiente para seu uso como controle no bloco teste utilizado, mesclaram-se pontos pré-sinalizados com pontos naturais bem visíveis na imagem de intensidade do levantamento laser e que puderam ser medidos com facilidade nas aerofotos. Cabe ressaltar que, durante o processo de coleta de pontos para a aerotriangulação na imagem de intensidade, observou-se a boa qualidade visual dos pontos présinalizados materializados por um quadrado branco e um círculo preto no centro. $\mathrm{O}$ grande contraste aparece muito claramente nos dois tipos de imagem (intensidade e aerofotos) como pode ser visto na Fig. 2. Tal propriedade merece atenção e apresenta um método interessante de integração entre aerofotos e dados laser scanner, podendo esse método ser associado futuramente a outros processos conhecidos de integração entre dados laser e imagens fotográficas digitais, como, por exemplo, através de feições lineares, conforme apresentado em (Habib et. al., 2004). 
Figura 2 - Pontos pré-sinalizados na Imagem de Intensidade (a) e nas Aerofotos (b).

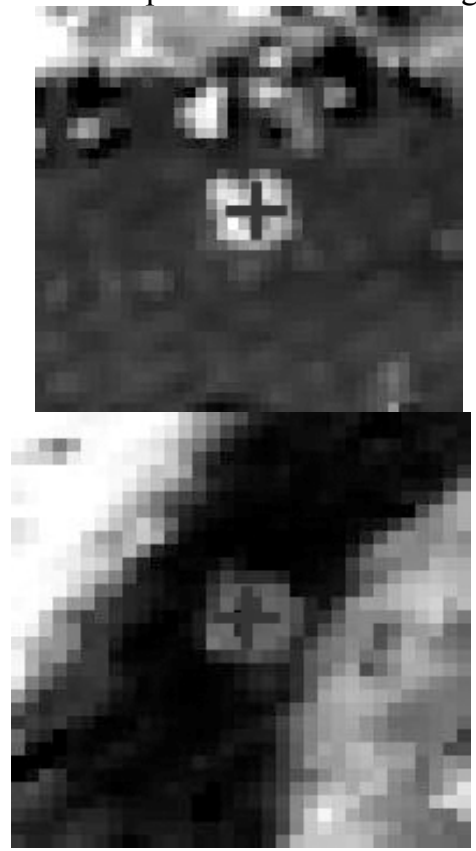

(a)
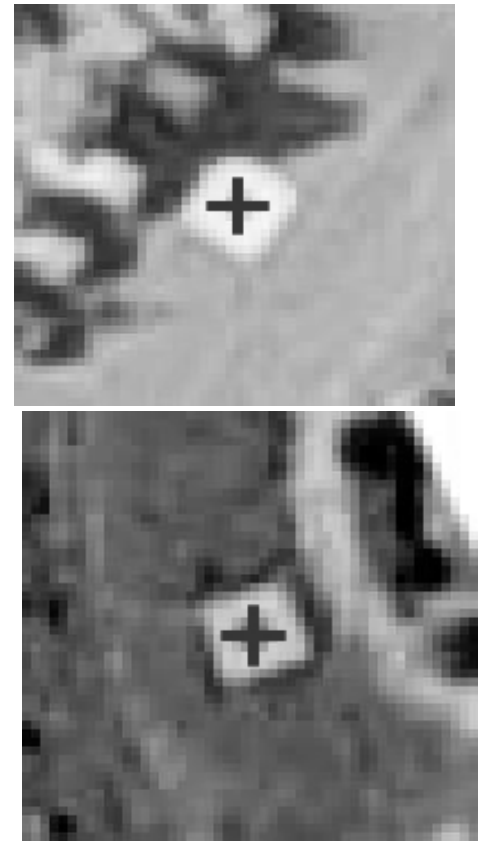

(b)

\section{RESULTADOS EXPERIMENTAIS}

Para se verificar a influência da calibração em serviço serão utilizados pontos de cheque na aerotriangulação, ou seja, pontos com coordenadas geodésicas conhecidas, as quais serão comparadas com as coordenadas obtidas pela aerotriangulação. Serão realizadas duas analises neste trabalho: uma através da comparação direta entre as coordenadas obtidas na aerotriangulação e as coordenadas geodésicas dos pontos de cheque; e outra análise da distribuição dos resíduos das observações no espaço-imagem dos ajustamentos realizados.

$\mathrm{Na}$ primeira análise, calculam-se as diferenças e realizam-se as análises estatísticas. Neste trabalho será realizado o teste " $\mathrm{t}$ " de Student para as médias das discrepâncias, de maneira semelhante à apresentada em trabalhos voltados à análise de precisão de cartas topográficas, como em Dalmolin et. al. (2001) e Galo et. al. (1994). Ambas as análises foram feitas a partir da aerotriangulação de um bloco de 13 imagens tomadas com uma câmera Sony DSC-F717 de 5.2 Mp de resolução e tamanho do pixel no solo (GSD) de aproximadamente $0,25 \mathrm{~m}$. Na Fig. (3) é apresentada a configuração do bloco. Destaca-se a foto 197, pois esta foi escolhida para uma analise dos resíduos do ajustamento (item 3.2). 
Partes dos dados utilizados nesse trabalho constam em (De Lara et. al., 2004). O recobrimento homogêneo e distribuição geométrica dos pontos fotogramétricos no bloco são necessários, para minimizar erros devidos a extrapolações, pois as equações matemáticas que envolvem os parâmetros adicionais, empregadas no refinamento das coordenadas fotogramétricas, são baseadas em polinômios.

Figura 3 - Disposição dos Pontos e Aerofotos no Bloco.

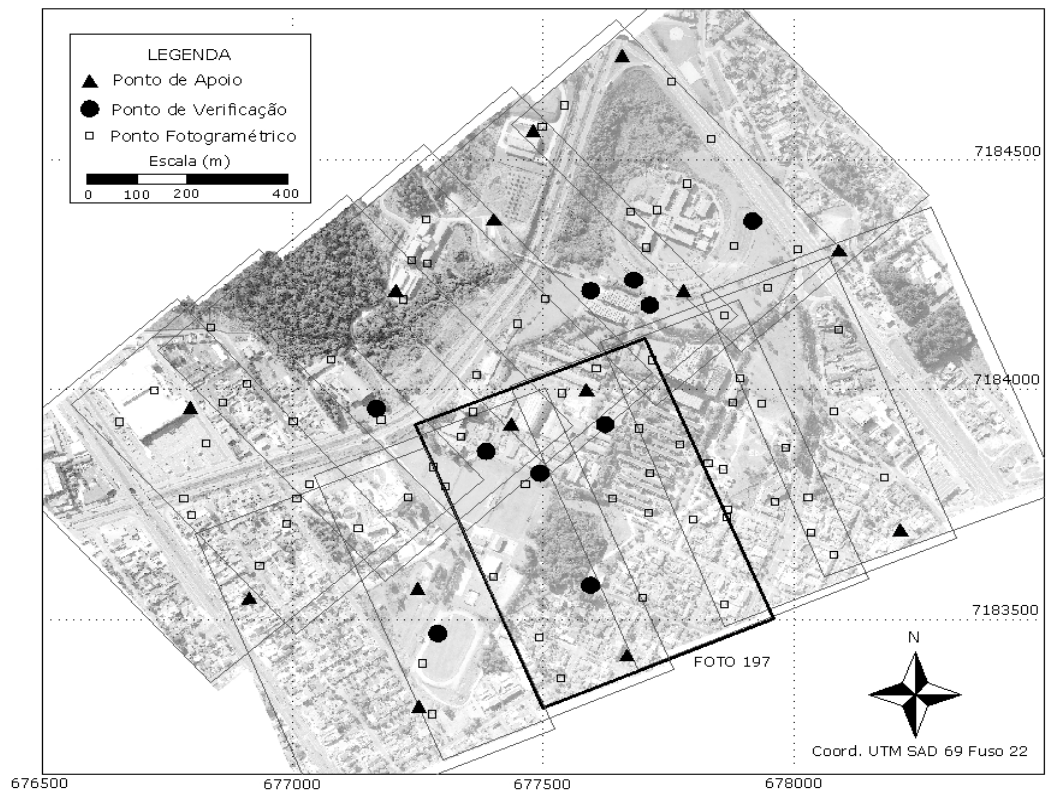

Para os pontos de apoio foi adotada a precisão de $0,25 \mathrm{~m}$ em $\mathrm{X}$ e $\mathrm{Y}$ (determinada com base no GSD) e de $0,15 \mathrm{~m}$ em Z, precisão aproximada das coordenadas do laser, pois os dados altimétricos possuem uma precisão melhor que a planimetria. A precisão adotada para as observações no espaço-imagem é de 0,5 pixel. A aerotriangulação foi realizada no referencial geodésico local, e as coordenadas finais transformadas para UTM para serem analisadas. Durante o processamento com auto-calibração foram eliminados os parâmetros adicionais $b_{1}$, $\mathrm{b}_{3}, \mathrm{~b}_{4}, \mathrm{~b}_{11}$ e $\mathrm{b}_{12}$; pois esses se mostraram não-significativos, ou seja não rejeitou-se a hipótese que o valor obtido fosse igual a zero, para 90\% de significância. Para 215 graus de liberdade (659 observações e 405 parâmetros), para um parâmetro ser considerado não-nulo o valor $|t|$ deve ser sempre superior a 1,256 . Verificou-se que retirando-se esses parâmetros do ajustamento, não ocorre uma variação significativa da a variância das observações de peso unitário a posteriori $\left(\sigma_{0}^{2}\right)$. A Tabela 1 
apresenta os valores obtidos para os parâmetros adicionais considerados significativos:

Tabela 1 - Parâmetros adicionais significativos.

\begin{tabular}{c|c|c|c}
\hline Parâmetro & Valor & Desvio Padrão & $|\mathbf{t}|$ \\
\hline $\mathrm{b}_{2}$ & $1,054853 \times 10^{-3}$ & $5,631075 \times 10^{-4}$ & 1,873 \\
\hline $\mathrm{b}_{5}$ & $-1,149767 \times 10^{-6}$ & $5,291227 \times 10^{-7}$ & 2,173 \\
\hline $\mathrm{b}_{6}$ & $-6,784402 \times 10^{-7}$ & $1,915882 \times 10^{-7}$ & 3,541 \\
\hline $\mathrm{b}_{7}$ & $-5,191415 \times 10^{-10}$ & $1,777683 \times 10^{-10}$ & 2,920 \\
\hline $\mathrm{b}_{8}$ & $-1,929325 \times 10^{-10}$ & $3,110714 \times 10^{-11}$ & 6,202 \\
\hline $\mathrm{b}_{9}$ & $3,731200 \times 10^{-10}$ & $1,770648 \times 10^{-10}$ & 2,107 \\
\hline $\mathrm{b}_{10}$ & $7,333639 \times 10^{-11}$ & $1,927014 \times 10^{-11}$ & 3,801 \\
\hline
\end{tabular}

\subsection{ANÁLISE DAS DISCREPÂNCIAS NO ESPAÇO OBJETO}

$\mathrm{Na}$ tabela 2 são apresentadas as médias $(\mu)$ e os desvios-padrão $(\sigma)$ das discrepâncias verificadas nas coordenadas dos 10 pontos de cheque. Apresentam-se também os valores " $\mathrm{t}$ " utilizados para a realização do teste de Student para a acurácia das coordenadas obtidas, conforme a equação (5), onde $\left(\mu_{0}\right)$ representa média esperada:

$$
t=\frac{\left(\mu-\mu_{0}\right)}{\sigma}
$$

Tal procedimento está descrito, por exemplo, em Dalmolin et. al (2001) e Galo et. al. (1994), e é adotado para testes de qualidade cartográfica com pequenas amostras. Adotando-se como média esperada $\left(\mu_{0}\right)$ o valor $0 \mathrm{~m}$, apresentam-se na Tab. 2 as estatísticas para os dois processamentos.

Tabela 2 - Estatísticas das Discrepâncias nos Pontos de Verificação.

\begin{tabular}{c|c|c|c|c|c|c}
\hline & \multicolumn{3}{|c|}{ Calibração em Serviço } & \multicolumn{3}{c}{ Clássico } \\
\hline & $\Delta \mathrm{E}$ & $\Delta \mathrm{N}$ & $\Delta \mathrm{h}$ & $\Delta \mathrm{E}$ & $\Delta \mathrm{N}$ & $\Delta \mathrm{h}$ \\
\hline$|\mu|$ & 0,177 & 0,105 & 0,064 & 0,238 & 0,192 & \\
\hline$\sigma$ & 0,206 & 0,254 & 0,168 & 0,329 & 0,386 & 0,273 \\
\hline$|\mathrm{t}|$ & 0,859 & 0,413 & 0,380 & 0,723 & 0,497 & 1,406 \\
\hline
\end{tabular}

Adotando-se como hipótese nula $\left(\mathrm{H}_{0}\right)$ que $\mu \leqslant \mu_{0}$, e hipótese não-nula $\left(\mathrm{H}_{1}\right)$ que $\mu>\mu_{0}$ e um nível de significância de $90 \%$, para o qual sabe-se que o valor $|t|$ deve ser menor que 1,38 (teste unilateral) para 9 graus de liberdade, como é o caso desta análise. Percebe-se claramente que tanto a planimetria quanto a altimetria 
passam no teste estatístico $\left(\mathrm{H}_{0}\right.$ não é rejeitada), quando da utilização da calibração em serviço. Na modelagem convencional, a altimetria não passa no teste.

Será realizada também uma análise gráfica dessas discrepâncias, para se verificar a presença de tendências sistemáticas, e tendo como base valores de tolerância calculados conforme a classe A do PEC (Padrão de Exatidão Cartográfica - decreto $n^{\circ}$ 89817). Para a escala 1:2000 (escala clássica de mapeamento cadastral urbano), esses valores são de $0,6 \mathrm{~m}$ para a planimetria e $0,5 \mathrm{~m}$ para a altimetria (considerando-se $1 \mathrm{~m}$ para a eqüidistância das curvas de nível). Na Fig. 4 é apresentada uma comparação entre os dois processamentos, o círculo representado aponta o valor da tolerância exigida pelo PEC. Na fig. 5 são representadas as discrepâncias ponto a ponto na altimetria para os dois processamentos:

Figura 4 - Comparação gráfica das discrepâncias em E e N.

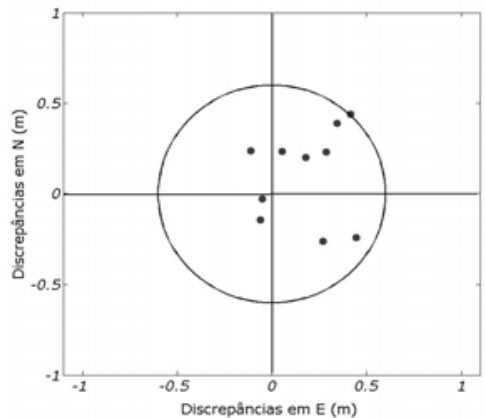

(a) Parâmetros Adicionais



(b) Convencional

Figura 5 - Comparação gráfica das discrepâncias em Z.

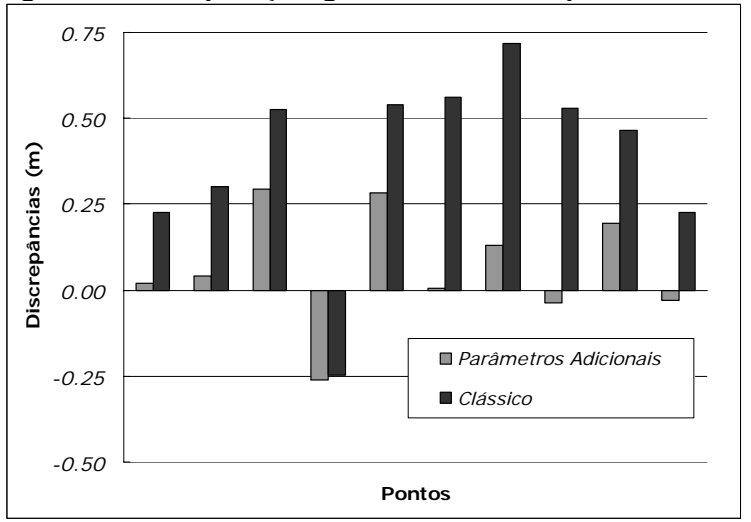

Bol. Ciênc. Geod., sec. Artigos, Curitiba, v. 16, nº 4, p.503-518, out-dez, 2010. 
A análise visual da fig. (4) permite concluir que há uma melhoria na distribuição das discrepâncias planimétricas quando da aplicação dos parâmetros adicionais. Observa-se uma pequena tendência, em ambos os casos, da coordenada E em direção Este. Para o processamento com parâmetros adicionais, todos os pontos estão dentro da tolerância e a distribuição das discrepâncias está homogênea. Já para a aerotriangulação convencional, alguns pontos estão fora da tolerância, mostrando uma pior distribuição dos erros. Observa-se claramente que na altimetria ocorre uma melhoria bastante significativa quando aplicados os parâmetros adicionais. Essa melhoria considerável na altimetria já tinha sido observada no teste estatístico das médias é prova de um melhor ajuste dos feixes de raios durante o processo de aerotriangulação.

Uma analise importante numa aerotriangulação é a da variância das observações de peso unitário a posteriori $\left(\sigma_{0}^{2}\right)$. Para o processamento com os parâmetros adicionais, obteve-se $\sigma_{0}{ }^{2}=0,85$ e no processamento clássico obteve-se $\sigma_{0}{ }^{2}=1,04$. A primeira conclusão importante é uma diminuição no $\sigma_{0}^{2}$ quando do uso de parâmetros adicionais. Isso indica uma diminuição dos resíduos na aerotriangulação. Dado que as discrepâncias em pontos de cheque são menores no processamento com parâmetros adicionais, pode-se afirmar que essa melhoria no $\sigma_{0}{ }^{2}$ é reflexo da qualidade das coordenadas finais obtidas e não por causa de uma superparametrização do ajustamento.

\subsection{ANÁLISE DA DISTRIBUIÇÃO DOS RESÍDUOS}

Será realizada a análise dos resíduos nas coordenadas fotogramétricas dos ajustamentos, os quais apontam possíveis erros sistemáticos nessas coordenadas, bem como possíveis tendências inseridas no sistema por causa da modelagem matemática utilizada, pois como pode ser observado nas eq. (2), o modelo matemático funcional do ajustamento é alterado quando da aplicação dos parâmetros adicionais, e essa alteração influencia diretamente as coordenadas fotogramétricas. Esse tipo de análise vem sendo utilizada no controle de qualidade das câmeras aerofotogramétricas digitais de grande formato, como pode ser visto, por exemplo em Dörstel (2007); e esta permite a verificação de padrões nos resíduos do ajustamento, como tendências radiais, tendências para uma determinada direção, fatores de escala, entre outros.

Para tal avaliação foi escolhida uma aerofoto (indicada na fig. 3), pois essa apresenta uma boa representatividade do bloco, com pontos de todos os tipos (apoio, cheque e fotogramétricos), bem distribuídos na imagem. Os resíduos, em pixel, foram plotados com um exagero de 400 vezes na fig. (6) a seguir: 
Figura 6 - Distribuição dos resíduos na aerofoto.

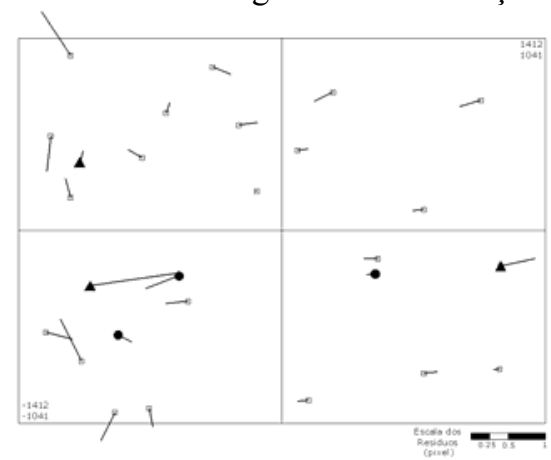

(a) Parâmetros Adicionais



(b) Convencional

Pode-se afirmar, comparando-se as direções dos resíduos dos processamentos, que a utilização dos parâmetros adicionais não provoca tendências sistemáticas no ajustamento, o que seria um indicativo de super-parametrização. Observa-se que, nos pontos de apoio (sinalizados com um triângulo), os resíduos são maiores no processamento com calibração em serviço do que na aerotriangulação convencional. Nos pontos de verificação e nos pontos fotogramétricos, o resíduo é maior no processamento clássico. A direção dos vetores é semelhante nos dois casos do processamento, indicando que a inclusão de parâmetros adicionais não insere tendências sistemáticas na aerotriangulação, se os parâmetros adicionais forem estatisticamente significativos.

Uma última analise estatística a ser realizada é a dos erros médios quadráticos (RMS) dos resíduos do ajustamento. Esses valores indicam a precisão obtida na aerotriangulação. Na Tab. 3 são mostrados os resultados para o RMS nos pontos de apoio do e na Tab. 4 o RMS dos resíduos nas coordenadas fotogramétricas:

Tabela 3 - RMS para os Resíduos nos Pontos de Apoio (m).

\begin{tabular}{c|c|c|c}
\hline Método & $\mathrm{E}$ & $\mathrm{N}$ & $\mathrm{Z}$ \\
\hline P. Adicionais & 0,336 & 0,339 & 0,180 \\
\hline Convencional & 0,506 & 0,413 & 0,231 \\
\hline
\end{tabular}

Tabela 4 - RMS para os Resíduos das Coordenadas Fotogramétricas (pixel).

\begin{tabular}{c|c|c}
\hline Método & $\mathrm{x}$ & $\mathrm{y}$ \\
\hline P. Adicionais & 0,391 & 0,293 \\
\hline Convencional & 0,444 & 0,332 \\
\hline
\end{tabular}


Os resultados acima vêm a corroborar com a hipótese de que há uma melhoria na precisão da aerotriangulação quando da aplicação dos parâmetros adicionais.

\section{CONCLUSÕES E RECOMENDAÇÕES}

Os resultados apresentados levam à conclusão de que a utilização dos parâmetros de calibração em serviço trazem melhorias aos resultados da aerotriangulação. Quando realizado o teste estatístico das médias, ambos os processamentos passam no teste planimétrico, contudo os resultados com parâmetros adicionais são melhores. Na altimetria o processamento com parâmetros adicionais passa no teste e o clássico não, mas o valor de $|t|$ ainda é aceitável, mesmo sendo mais alto que $\mathrm{o}|\mathrm{t}|$ obtido com a adição dos parâmetros adicionais. $\mathrm{Na}$ análise gráfica das discrepâncias altimétricas, observa-se um melhor desempenho do método com parâmetros adicionais. Esses resultados levam à conclusão que, para um usuário final (como, por exemplo, para a realização de restituição fotogramétrica), o resultado da aerotriangulação convencional é suficiente. No entanto, se os resultados da aerotriangulação forem utilizados como etapa intermediária num processamento mais sofisticado, no qual possa ocorrer uma maior propagação de erros, faz-se necessário uma melhor modelagem matemática, e a calibração em serviço se mostra como uma solução viável.

Um problema recorrente na introdução de parâmetros adicionais numa aerotriangulação é a possibilidade de se ocorrer super-parametrização no ajustamento, ou seja, um falseamento dos resultados. Kraus (1996) afirma que o número de parâmetros adicionais deve ser o menor possível. Testes realizados nos pontos de verificação mostraram que os melhores resultados na variância da unidade de peso a posteriori $\left(\sigma_{0}^{2}\right)$ não são causados por super-parametrização, mas por uma melhor modelagem matemática. No teste dos resíduos das coordenadas fotogramétricas observou-se um comportamento semelhante nos dois processamentos realizados. Tomando-se a aerotriangulação convencional como isenta de influências sistemáticas, e tendo os dois processamentos um comportamento semelhante, pode-se afirmar que a introdução dos parâmetros de calibração em serviço não provoca super-parametrização desde que os parâmetros determinados sejam significativos. Como trabalho futuro, propõe-se uma cuidadosa análise na correlação entre os parâmetros adicionais. Outro resultado obtido, através da determinação dos erros médios quadráticos, é que a precisão geral do ajustamento melhora quando da utilização dos parâmetros adicionais. Tendo em vista esses fatores, pode-se concluir que a utilização conjunta dos parâmetros de calibração em serviço com os convecionais é uma metodologia viável para se compensar erros não modelados pela calibração convencional. 


\section{AGRADECIMENTOS}

Os autores desejam expressar seus agradecimentos à CAPES (Coordenação de Aperfeiçoamento de Pessoal de Nível Superior) pela bolsa de doutorado que possibilita a realização deste trabalho.

\section{REFERÊNCIAS BIBLIOGRÁFICAS}

ACKERMANN, F. Block Adjustment with Additional Parameters. Photogrammetria, 36 (1981). pp. 217-227.

BROWN, D.C. Advanced Methods for the Calibration of Metric Cameras. In: ISPRS Symposium on Computational Photogrammetry, Syracuse University, January 1969. Proceedings. 1969.

BROWN, D.C. A strategy for multi-camera on-the-job self-calibration. Institut für Photogrammetrie Stuttgart, Festschrift, Friedrich Ackermann, zum 60. Geburtstag, 13 pages.

CLARKE, T.A; FRYER, J.F. The development of camera calibration methods and models. The Photogrammetric Record, 16(91): pp 51-66. 1998.

CRAMER, M. Calibration and validation of digital airborne cameras. In: ISPRS Commission I Symposium "From Sensors to Imagery", Paris, July 4-6. 2006. Proceedings. 2006

DALMOLIN, Q.; LEAL, E. M. Análise da qualidade posicional em bases cartográficas geradas em CAD. Boletim de Ciências Geodésicas, Curitiba, v. 7, n. 1, p. 21-40, 2001.

DE LARA, R.; MITISHITA, E.; HABIB, A. Bundle adjustment of images from non-metric CCD camera using LIDAR data as control points. International Archives of XXth ISPRS Congress 2004, Istambul, Turkey, Comission III, 2004, p. 13-19.

DE LARA, R.; MITISHITA. E.; BÄHR H-P.; VÖGTLE., T. Metodologia para integração automática de imagens aéreas digitais e dados SPLA. Boletim de Ciências Geodésicas, Curitiba, v. 13, n. 2, p. 369-394, 2007.

DÖRSTEL, C. DMC - (R)evolution on Geometric Accuracy. Photogrammetric Week '07, Wichmann, p. 81-88. 2007.

EBNER, H. Self calibrating block adjustment. International Archives of XIIth ISPRS Congress 1976, Helsinki, Finland, Invited Paper of Comission III, 1976.

GALO, M.; CAMARGO, P. O uso do GPS no controle de qualidade de cartas. In.: COBRAC-1994, $1^{\circ}$ Congresso Brasileiro de Cadastro Técnico Multifinalitário. Tomo II, p. 41-48, Florianópolis, 1994

GEMAEL, C. Introdução ao ajustamento de observações. Aplicações geodésicas. Editora UFPR, Curitiba, 1994, 319p.

GRÜN, A. Progress in Photogrammetric Point Determination by Compensation of Systematic Errors and Detection of Gross Errors. Nachrichten aus dem Karten- und Vermessungswesen. Series II, 36, 113-140. 1978. 
HABIB, A; MORGAN, M; LEE, Y. Bundle Adjustment with Self-Calibration Using Straight Lines. The Photogrammetric Record, v. 17, n.100, p. 635-650, October. 2002.

HABIB, A.; GHANMA, M.; MITISHITA, E. Co-registration of photogrammetric end LIDAR data: methodology and case study. Revista Brasileira de Cartografia n. 56/01, 2004.

HABIB, A.; MORGAN, M.. Stability analysis and geometric calibration of off-theshelf digital cameras. Photogrammetric Engineering \& Remote Sensing, 71(6): 733-741. 2005

HABIB, A.; PULLIVELLI, A.; MITISHITA, E.; GHANMA, M.; KIM, E. Stability analysis of low-cost digital câmeras for aerial mapping using different georeferencing techniques. The Photogrammetric Record. v.21 n.113 pp. 2943, March 2006.

JACOBSEN, K. Programmgesteuerte Auswahl zusaetzlicher Parameter. Bildmessung und Luftbildwesen, v. 50, pp. 213-217. 1982.

JACOBSEN, K. Direct Georeferencing. Photogrammetric Engineering and Remote Sensing, 67(12), 2001.

JUBANSKI, J.; ALIXANDRINI, M.; BÄHR, H-P. Câmeras Aerofotogramétricas Digitais: Novos Conceitos e Normas. Revista Brasileira de Cartografia n. 61/01, 2009.

KUPFER, G.; MAUELSHAGEN, L. Correlations ans Standard Errors in Bundle Block Adjustment with Some Emphasis on Additional Parameters. Photogrammetria 38, pp. 57-72, 1982.

LÄBE, T.; FÖRSTNER, W. Geometric Stability of low-cost Digital Consumer Cameras. In: International Archives of Photogrammetry, Remote Sensing and Spatial Information Sciences. n. 35(5) pp. 528-535, 2004.

KRAUS, K. Photogrammetrie. Band 2: Verfeinerte Methoden und Anwendungen. Ferd. Dümmlers Verlag, Bonn, 1996, 494p.

MACHADO, A. Extração Automática de contornos de edificações utilizando imagem gerada por câmera digital de pequeno formato e dados LIDAR. Curitiba, 2006. Tese (Doutorado), Curso de Pós-Graduação em Ciências Geodésicas, UFPR.

MARCATO, J.; TOMMASELLI, A.; RUY, R.; GALO, M. Estudos de caso em Fototriangulação com Parâmetros Adicionais para Câmeras Digitais. In: Anais do Congresso Brasileiro de Cadastro Técnico Multifinalitário (COBRAC). 2008,

MONIWA, H. The Concept of "Photo-Variant"Self Calibration and its Application in Block Adjustment with Bundles. Photogrammetria, 36, pp. 11-29, 1981.

WANDRESEN, R. Integração entre neurais e correlação para identificar pontos de apoio pré-sinalizados. Curitiba, 2004. Tese (Doutorado), Curso de PósGraduação em Ciências Geodésicas, UFPR.

(Recebido em agosto de 2008. Aceito em setembro de 2010.) 